\title{
Hippocampal Activations during Repetitive Learning and Recall of Geometric Patterns
}

\author{
Georg Grön, ${ }^{1}$ Daniel Bittner, ${ }^{3}$ Bernd Schmitz, ${ }^{3}$ Arthur P. Wunderlich, ${ }^{2}$ \\ Reinhard Tomczak, ${ }^{2}$ and Matthias W. Riepe ${ }^{3,4}$ \\ ${ }^{1}$ Department of Psychiatry, University of Ulm, Leimgrubenweg 12, 89075 Ulm, Germany; ${ }^{2}$ Department of Radiology, University of Ulm, \\ Steinhövelstr. 9, 89075 Ulm, Germany; ${ }^{3}$ Department of Neurology, University of Ulm, Steinhövelstr. 1, 89075 Ulm, Germany
}

\begin{abstract}
Hippocampal activation is required for episodic memory. Encoding and retrieval of novel and memorable items have been related to different locations in the hippocampus; however, the data remain ambiguous. The application of a newly designed keyboard allowed investigation of brain activation during encoding and free immediate and delayed recall with functional magnetic resonance imaging (fMRI) in young healthy controls $(n=12)$. Because of the repetitive learning and recall conditions, an individual learning gradient was used to contrast neural activity at different individual levels of novelty. During learning, subjects were asked to memorize 10 geometric patterns requiring the establishment of intra-item associations for memorization. After learning, subjects were asked to recall the items actively via the keyboard. Learning and recall were alternated five times. Delayed recall was scanned about $15 \mathrm{~min}$ after the fifth immediate recall condition without subjects having seen the items again. Left-sided anterior hippocampal activity was observed during conditions of initial learning as well as maximum recall. Neural activity during delayed recall did not reveal hippocampal responses and was characterized by a transition of neural activity from occipitoparietal regions to bilateral temporal cortices. We conclude that both lateralization and segregation depend on the specific relational characteristics of the stimuli requiring establishment of intra-item associations for encoding as well as retrieval. The absence of hippocampal activation during delayed recall together with the increase of lateral temporal involvement possibly corresponds with an emerging transition from episodic to long-term memory.
\end{abstract}

Neuropsychological (Milner et al. 1998; Vargha-Khadem et al. 1997) and animal studies (Wood et al. 1999) clearly show the importance of the integrity of the hippocampus proper for episodic memory. From the discussion on functional imaging of hippocampal structures during episodic memory tasks (e.g., Fletcher et al. 1995; Buckner and Koutstaal 1998; Fernandez et al. 1998; Schacter and Wagner 1999) it can be concluded that observance of hippocampal activity may correspond to such factors as the modality of the stimuli (verbal vs. visual) and/or the retrieval process engaged (recall vs. recognition). Whereas verbal stimuli may not reliably elicit hippocampal responses (Shallice et al. 1994; Buckner et al. 1995), unusual or complex visual stimuli did ( Roland and Gulyas 1995; Maguire et al. 1996; Stern et al. 1996; Gabrieli et al. 1997). The latter stimuli usually consist of multiple inputs and permit representations of the relations among the constituent elements (Cohen et al. 1999). The retrieval process appears to be critical for observance of hippocampal activity whether the task requires recognition or active recall (recollection). From studies on recognition and recall in amnesia it was concluded that recogni-

${ }^{4}$ Corresponding author.

E-MAIL matthias.riepe@medizin.uni-ulm.de; FAX 49731502 6745.

Article and publication are at http://www.learnmem.org/cgi/doi/ $10.1101 / \mathrm{lm} .42901$. tion involves the perirhinal cortex of the temporal lobe, whereas recollection depends on the hippocampus (Aggleton and Brown 1999), which is also consistent with recent neuroimaging studies (Henson et al. 1999; Cabeza et al. 2000; Eldridge et al. 2000; but see also Stark and Squire 2000)

More recent imaging studies that observed hippocampal activation (for review, see Lepage et al. 1998) support another discussion on hippocampal activity. Data from experimental animals (Moser and Moser 1998) and lesion studies on humans (Phelps et al. 1991; Corkin et al. 1997) indicated a functional segregation of memory functions in the hippocampus. It was concluded that the anterior hippocampal formation is relevant for encoding (novelty-related processing), whereas the posterior hippocampal sections are responsible for familiarity-related processing and retrieval demands (Dolan and Fletcher 1999). A recent meta-analysis of positron emission tomography (PET) studies on hippocampal activation during episodic memory (Lepage et al. 1998) revealed that most of the PET studies were very consistent with these predictions. Additionally, a recent functional magnetic resonance imaging (fMRI) study on the involvement of medial temporal regions during memory processing of words showed that novelty processing activated the anterior hippocampus, whereas processing of familiar words was linked to the posterior hippocampal formation

LEARNING \& MEMORY 8:336-345 @ 2001 by Cold Spring Harbor Laboratory Press ISSN1072-0502/01 \$5.00

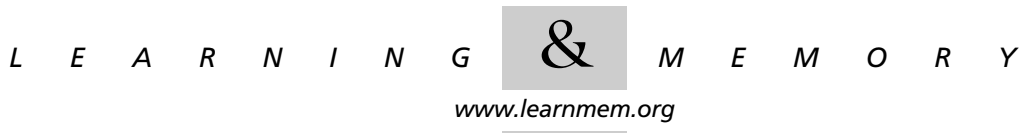


(Saykin et al. 1999). However, exactly the opposite conclusions were drawn from reviewing recent fMRI studies (Schacter and Wagner 1999) as well as from a PET study that directly addressed this issue (Schacter et al. 1999).

Current understanding of memory consolidation postulates that episodic learning initially requires the hippocampal formation, but gradually memories become independent from this structure and are established in distributed neocortical storage sites (Alvarez and Squire 1994; Graham and Hodges 1997; Graham et al. 1999). However, other recent evidence indicates that the hippocampal formation is always necessary for episodic memory (Nadel and Moscovitch 1997; Nadel et al. 2000). If the latter hypothesis is correct, episodic long-term retrieval of the previously learned stimuli should be correlated with hippocampal activity.

The goals of the current study were to investigate hippocampal activity during encoding and active immediate recall of abstract visual geometric designs, address functional segregation thereof with respect to different levels of novelty during encoding and retrieval, and study delayed recall after a longer interval.

\section{RESULTS}

\section{Behavioral Performance}

Performance (mean and standard deviations of correctly recalled figures) during scanning is shown in Figure 1. A multivariate analysis of variance (MANOVA) for repeated measures revealed a significant effect of repetitive recall conditions on mean correct performance $(\mathrm{F}(4,8)=57.81$; $P<.001$ ). Post-hoc Newman-Keuls tests (nominal level of alpha: $P<.05)$ showed significant increases in performance from the first to the second recall condition as well as from the second to the third repetition. Performance did not significantly increase from the third to the fourth repetition

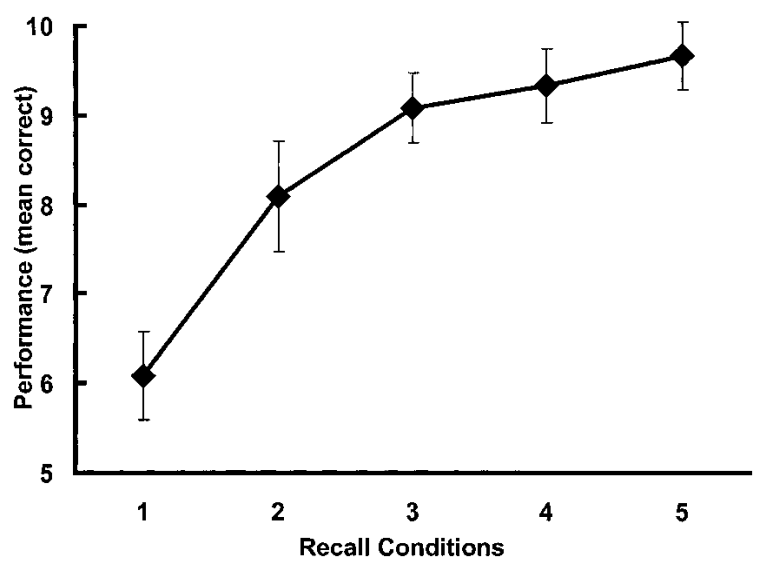

Figure 1 Performance during scanning. The graph shows the mean and standard deviations of correctly recalled geometric patterns over the five repetitive recall conditions. or from the fourth to the fifth recall condition. Accordingly, polynomial contrasts within a MANOVA for repeated measures showed a better fit for the logarithmic trend of the learning function $(\mathrm{F}(1,11)=169.0, P<.001$; explained variance: $96.8 \%)$ than for the linear trend $(F(1,11)=144.0$; $P<.001$; explained variance: $84.4 \%$ ). For most of the subjects ( 7 of 12) the individual maximum recall was reached at the fourth recall phase. Taken as a group, the mean number of phases in which maximum recall was reached was 4.0 (SD: \pm 0.95 ). Recall performances between the fifth and sixth recall phase (mean correct: $9.6 \pm 0.4$ ) did not significantly differ $(\mathrm{t}(11)=1.15, P=.27)$.

\section{Functional Imaging}

Group results for the planned contrasts (initial/late learning; initial/maximum recall) are summarized in Table 1 . Neural activity during initial learning was mainly characterized by the left prefrontal areas, the left parahippocampal gyrus, and the anterior portion of the left hippocampus proper (Fig. 2A). On the right hemisphere two further regions were involved in initial learning: the fusiform gyrus and the anterior cingulate. For late learning, mid-parietal regions were active (Fig. 2B). Neural activity during initial recall was observed in the left frontal areas, the right occipital areas, and the right posterior hippocampal area (Fig. 2C). Group activity of maximum recall revealed superior, inferior, and mid-parietal regions on both hemispheres. Moreover, the precentral gyrus and the cingulate gyrus yielded significant activations. Activity of the hippocampus proper was left lateralized (Fig. 2D)

To explore the hippocampal activity during initial learning, initial recall, and maximum recall at various locations along the longitudinal axis of the hippocampus, two masks comprising the left and right hippocampus, respectively, were constructed (for definition of borders, see Pruessner et al. 2000). These masks were applied on the $t$-maps resulting from the one-sample $t$-tests mentioned above. Maximum $t$-values from each of the 23 left and right coronal slices comprising the longitudinal axis of the hippocampus in anterior-posterior direction were determined for each condition separately. Thus, Figures 3A and B represent gradients of group activations along the long axis of the hippocampus based on the most significant voxel per slice. With respect to the left hippocampus, activity for initial learning increased with anterior slices and remained significant (Fig. 3A, 19 successive slices from slices 3-22) with peak activity in the anterior half of the long axis (slice 11). In contrast, peak activity for maximum recall is located in the seventh slice, that is, about $8 \mathrm{~mm}$ more anterior than for initial learning, and decreased more rapidly than during initial learning, thus comprising only nine successive slices from anterior to posterior (slices $4-13$ ). Peak activity for initial recall is at approximately the same location as it is for initial learning, although lowered. On the right hemisphere,

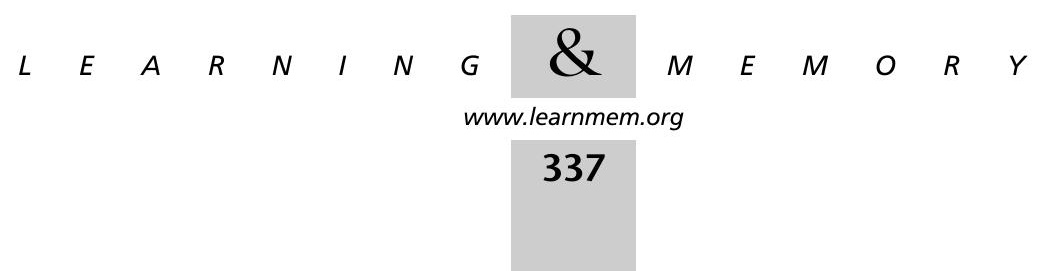


Table 1. Anatomical Regions Activated During Initial/Late Learning and Initial/Maximum Recall

\begin{tabular}{|c|c|c|c|c|c|c|c|c|c|c|c|}
\hline \multicolumn{6}{|l|}{ Initial learning } & \multicolumn{6}{|c|}{ Late learning } \\
\hline Left & BA & $\mathrm{x}$ & $y$ & z & z-value & Left & BA & $\mathrm{x}$ & $y$ & z & z-value \\
\hline Hippocampus, anterior & & -24 & -16 & -14 & 3.68 & Precuneus & 7 & -4 & -70 & 38 & 3.69 \\
\hline Parahippocampal gyrus & 35 & -12 & -8 & -14 & 3.28 & & & & & & \\
\hline Dorsal frontal gyrus & $9 / 10$ & -6 & 44 & 18 & 3.32 & & & & & & \\
\hline Middle frontal gyrus & 9 & -32 & 10 & 36 & 2.94 & & & & & & \\
\hline Right & & & & & & Right & & & & & \\
\hline Fusiform gyrus & 36 & 34 & -44 & -22 & 4.22 & Precuneus & 7 & 0 & -34 & 50 & 3.65 \\
\hline Anterior cingulate gyrus & 24 & 16 & 14 & 36 & 3.97 & Posterior cingulate gyrus & 23 & 2 & -22 & 26 & 3.65 \\
\hline \multicolumn{6}{|c|}{ Initial recall } & \multicolumn{6}{|c|}{ Maximum recall } \\
\hline Left & BA & $\mathrm{x}$ & y & z & z-value & Left & BA & $x$ & y & z & $z$-value \\
\hline \multirow[t]{6}{*}{ Middle frontal gyrus } & 9 & -46 & 12 & 38 & 3.25 & Superior parietal lobule & 7 & -22 & -50 & 68 & 4.03 \\
\hline & 9 & -24 & 36 & 36 & 3.12 & Hippocampus, anterior & & -32 & -14 & -14 & 3.64 \\
\hline & & & & & & Anterior cingulate gyrus & 6 & -10 & 6 & 38 & 3.42 \\
\hline & & & & & & Precentral gyrus & 6 & -60 & -6 & 36 & 3.38 \\
\hline & & & & & & Inferior parietal lobule & 40 & -62 & -22 & 24 & 3.04 \\
\hline & & & & & & Superior temporal gyrus & 42 & -52 & -28 & 18 & 2.92 \\
\hline Right & & & & & & Right & & & & & \\
\hline Fusiform gyrus & 37 & 30 & -52 & -8 & 4.11 & Precuneous & 7 & 4 & -46 & 60 & 3.75 \\
\hline Hippocampus, posterior & & 30 & -42 & -2 & 3.38 & Inferior parietal lobule & 40 & 52 & -20 & 30 & 3.58 \\
\hline Cuneus & 31 & 20 & -66 & 16 & 3.21 & Superior temporal gyrus & 22 & 62 & -24 & 20 & 2.85 \\
\hline
\end{tabular}

(BA) Brodmann's areas, $x, y, x$ : stereotaxic co-ordinates. For calculation of contrasts, refer to text.

graphs for initial learning and maximum recall represent the same relationships, although peak activity is generally reduced when compared with the left side. For initial recall, only the posterior slices crossed the critical threshold.

For delayed recall (Fig. 4A) we observed almost exclusively strong bilateral activations of the temporal cortices: left superior temporal gyrus (BA 22 maximum peak at $\mathrm{x}=-46, \mathrm{y}=-42, \mathrm{z}=16 ; Z$-value $=4.48 ; \mathrm{BA} 42$ maximum peak at $\mathrm{x}=-48, \mathrm{y}=-10 \mathrm{z}=10 ; Z$-value $=4.08)$, left middle temporal gyrus (BA 21 maximum peak at $\mathrm{x}=-44, \mathrm{y}=-20$, $\mathrm{z}=-8 ; Z$-value $=4.09)$, right middle temporal gyrus $(\mathrm{BA} 21$, maximum peak at $\mathrm{x}=50, \mathrm{y}=-32, \mathrm{z}=0 ; Z$-value $=3.88)$, and right inferior temporal gyrus (BA20, maximum peak at $\mathrm{x}=62, \mathrm{y}=-34, \mathrm{z}=0 ; Z$-value $=3.74)$. Additionally, activation of the precuneus (BA 31, maximum peak at $x=6$, $\mathrm{y}=-70, \mathrm{z}=20 ; Z$-value $=4.25$ ) was elicited. We did not observe any hippocampal activity even when lowering the statistic voxel thresholds to a level of $P<.05$.

To summarize the pattern with respect to temporal and hippocampal activity across early to late recall, effect sizes at corresponding voxels demonstrating maximum peak activity for initial, maximum, and late recall at hippocampal and temporal locations were determined for each subject. In cases with multiple temporal peak activities, effect sizes were averaged irrespective of laterality. The results are in Figure $4 \mathrm{~B}$ showing an interaction of time by location.

\section{DISCUSSION}

The new paradigm of repetitive learning and recall of abstract geometric designs showed robust hippocampal re- sponses. Right posterior hippocampal activation was observed when initial recall was contrasted against individual maximum recall. Clusters of left anterior hippocampal activity were observed during initial learning and maximum recall. No hippocampal activity was observed during late learning or delayed recall.

\section{Lateralization}

At first glance, our data are counterintuitive with respect to the left-sided lateralization of hippocampal responses during initial learning and maximum recall. Because of the visuospatial characteristics of the material presented, one may have expected additional right-sided hippocampal activity (Bohbot et al. 1998). The lateralization to the left side could suggest that there are language-related processes engaged during the initial learning or maximum recall of the geometric patterns. This would be in line with a recent review on automatic activation of the medial temporal lobe (MTL), especially during encoding (Martin 1999). Summarizing a series of functional brain-imaging studies, it was concluded that MTL activity is left lateralized when verbal material is to be processed and right lateralized when nonverbal material is presented. With deep encoding, which was argued to be equivalent to encoding for meaning, leftlateralized language mechanisms are activated (Martin 1999). Although we cannot completely rule out languagerelated processing, we did not observe activation of other left cortical areas associated with language processing, such as the supramarginal gyrus, Broca's area, the insula, or areas within the left inferior frontal lobe. These regions are

$$
\begin{array}{lllllllllllllll}
L & E & A & R & N & I & N & G & \mathcal{Q} & M & E & M & O & R & Y \\
\text { www.learnmem.org } & & & & &
\end{array}
$$




\section{A Initial learning minus late learning}

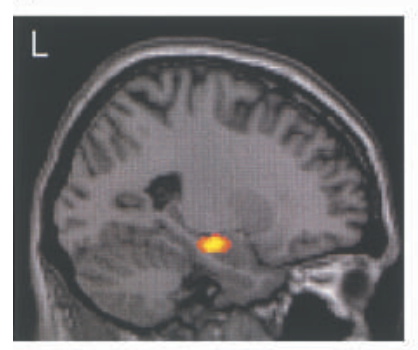

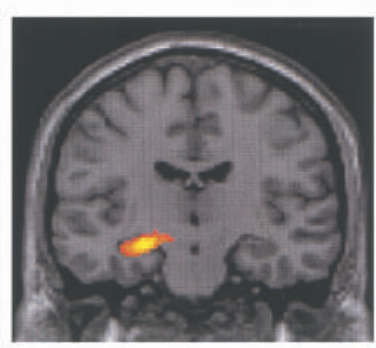

\section{B}

Late learning minus initial learning
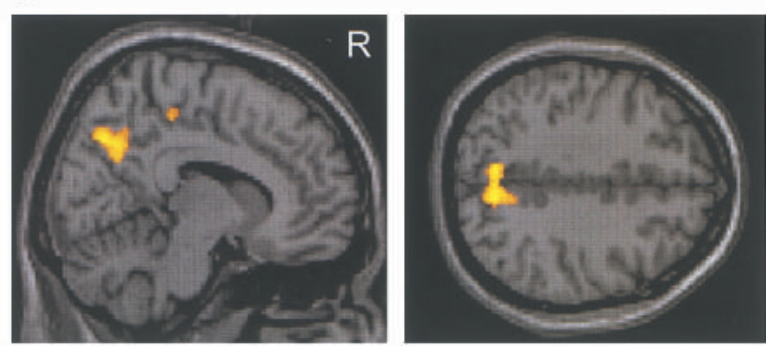

\section{Initial recall minus maximum recall}

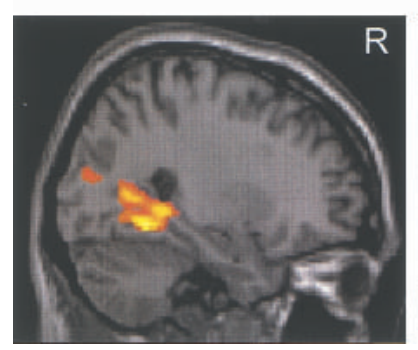

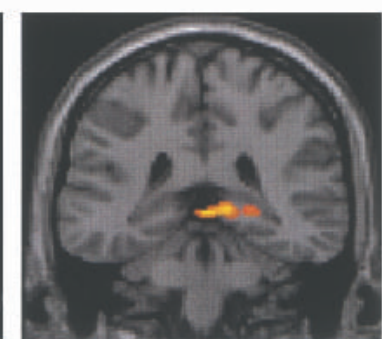

\section{Maximum recall minus initial recall}

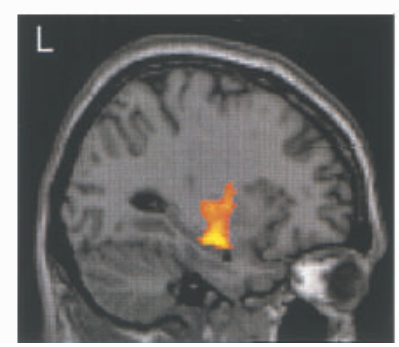

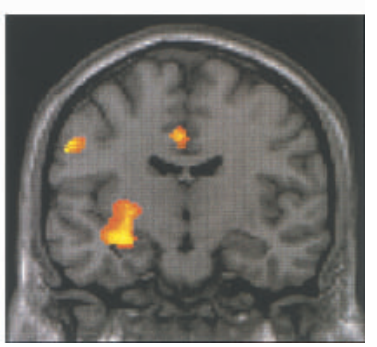

Figure 2 Functional imaging data superimposed on a $\mathrm{T}_{1}$-weighted image in standardized space. (A) Activity of the left anterior hippocampal region during initial encoding. (B) Activity of the mid-parietal areas during late learning. (C) Activity of the right posterior hippocampal region (parahippocampus/hippocampus) during initial recall that is not further discernible at the present resolution imposed by voxel size and kernel used in this study. $(D)$ Activity of the left anterior hippocampus region during maximum recall. Taking activity around the peak into account, we also observed activations including caudal parts of putamen $(x=-28, y=-6, z=2 ; Z$-value $=3.06)$ and claustrum $(x=-34, y=0, z$ $=14 ; Z$-value $=2.88$.

known as constituting the phonological or articulatory loop (Paulesu et al. 1993; Paulesu et al. 1996). If encoding by the subjects was based on language, then these areas should have been additionally engaged, especially during an initial and intentional encoding of the presented patterns. Beyond this, the exact structure of the stimuli seems to be critical for engaging neural substrate unilaterally or bilaterally. Specifically, abstract geometric patterns that are constituted by spatial relations of their elements have been shown not to have lateralization preference in humans (Sergent 1991) and monkeys (Hamilton and Vermeire 1988). In addition, there are numerous imaging studies reporting left-sided hippocampal activity during the encoding of figures or relational environmental information (Aguirre et al. 1996; Brewer et al. 1998; Kelley et al. 1998; Montaldi et al. 1998; Stern et al. 1996). In a study on navigation through threedimensional mazes (Grön et al. 2000), it was concluded from left hippocampal activity in men that this was functionally related to the processing of relational topographic information, allowing men a faster and more successful navigation than women who did not engage the left hippocampus. A lack of lateralization has been reported for verbal episodic memory (Dobbins et al. 1998), and a recent study on learning, retention of words, and abstract designs after unilateral excision of MTLs could not confirm a double dissociation between the kind of material and the site of excision. The results for learning and retention of abstract designs were not exclusively confined to the right resection group (Jones-Gotman et al. 1997). Additionally, divided visual field experiments in normal subjects provided a left hemispheric advantage for the processing of categorical spatial relations that contrasted with a right superiority for metric judgments of distances between two objects (Kosslyn et al. 1989). Therefore, lateralization of the hippocampal activity may not be specific to the material but rather to the combination of material and task demands.

\section{Functional Segregation}

For initial learning, cluster-analysis revealed an anterior locus of hippocampal activity (Fig. 2A). Tracking the most significant voxels along the hippocampal long axis for this contrast, (Fig. 3A) activation is extended along the hippocampus. Clear-cut anterior predominance is observed during maximum recall, as revealed by both kinds of analyses (Figs. 2C and 3A). For initial recall, that is, condition of low

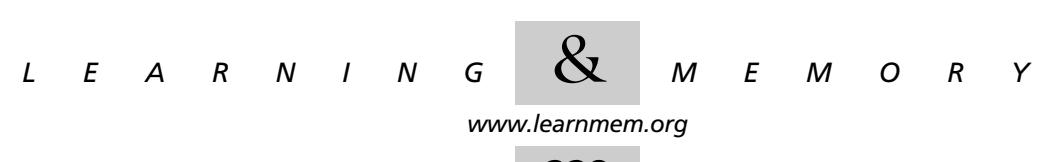


A

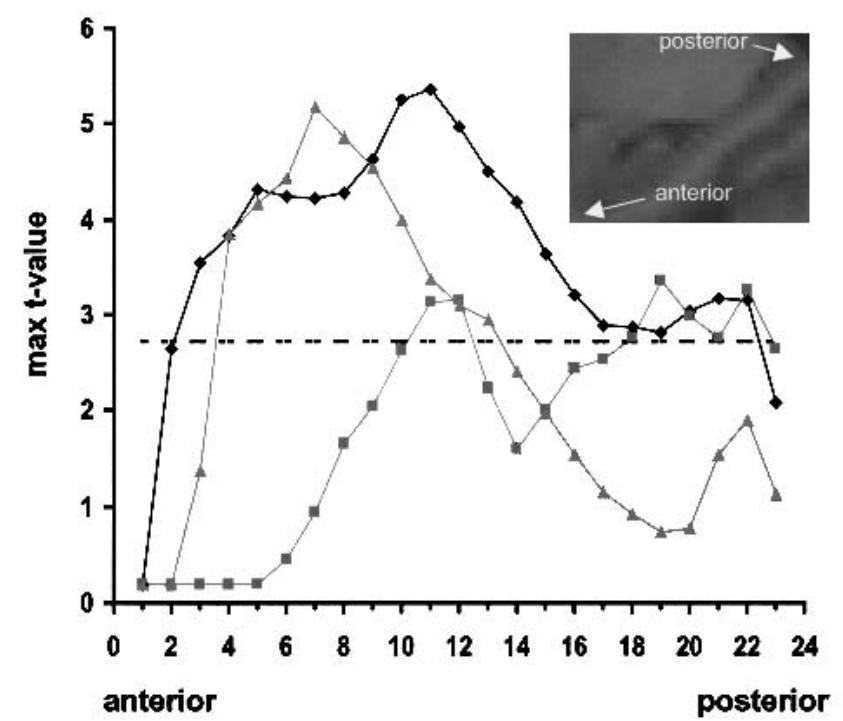

B

Right hippocampus

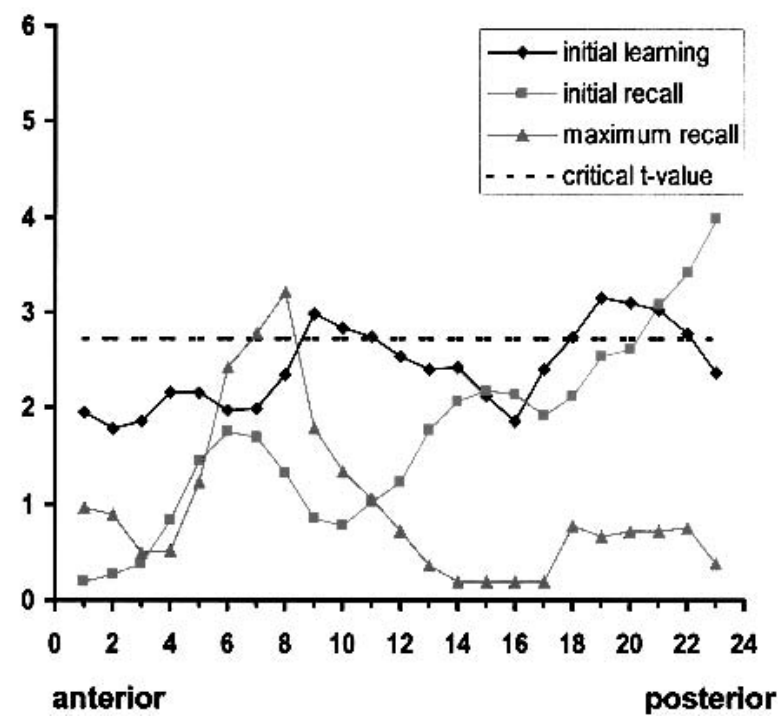

Figure 3 Graphs represent the maximum $T$-values of corresponding voxels from each of the 23 coronal slices comprising the longitudinal axis of the hippocampus. The height of the critical $T$-value is represented by the dashed line. Values above this line are significant $(P<.01)$. (A) Left hippocampus, $(B)$ right hippocampus.

memorability, cluster and voxel-based analyses consistently showed hippocampal activity that was located posteriorly.

Peak activities for initial learning and maximum recall were $\sim 8 \mathrm{~mm}$ apart but still in the anterior half of the long axis of the hippocampus. Thus, the current results only in part reproduce the opposing conclusions of both conflicting recent studies (Lepage et al. 1998; Saykin et al. 1999).

The conflicting conclusions can be resolved by an alternative explanation. When analyzing the different results of PET and fMRI studies on hippocampal activity along the

\section{A Delayed recall minus initial recall}

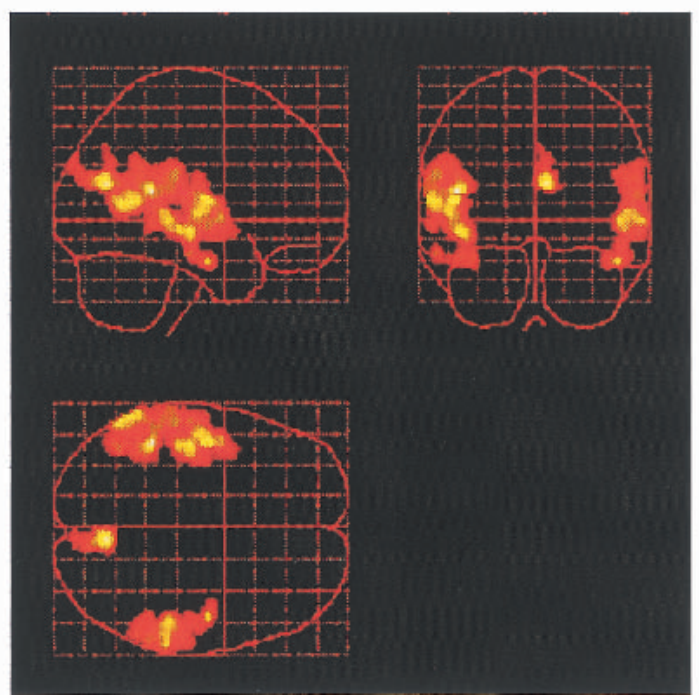

B

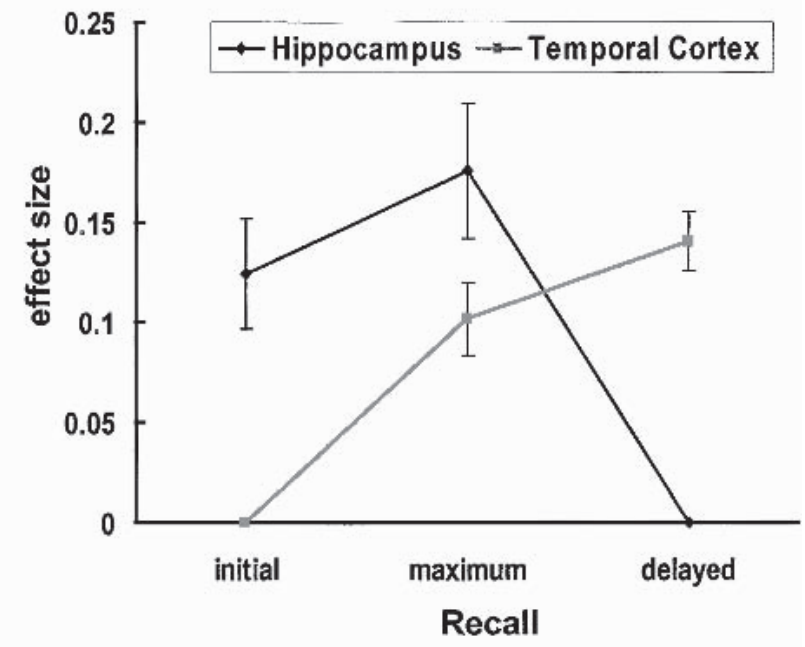

Figure 4 (A) Bilateral temporal activations during long-term recall on sagittal, coronal, and transverse views of a glass brain. (B) Location by time interaction across initial to delayed recall. ANOVA for repeated measures revealed a significant effect of time $(F(2,44)=8.178$; $P=.0006)$ but no significant effect of location $(F(1,22)=1.373 ; P=.254)$. The interaction of time by location was highly significant $(\mathrm{F}(2,44)=24.247 ; P<.001)$.

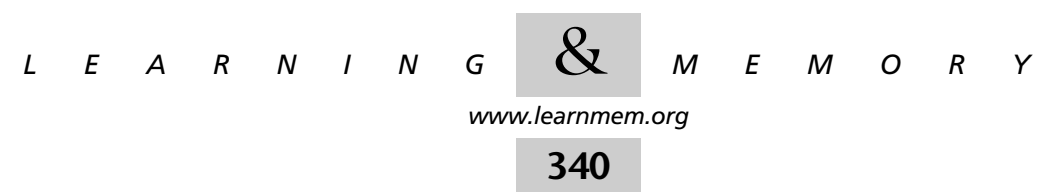


anterior-posterior axis during encoding, it was pointed out that five of the PET studies that observed anterior hippocampal activity during encoding required the participants to focus on the relations among multiple stimuli (Schacter and Wagner 1999). This was also the case in an fMRI study (Rombouts et al. 1997) that yielded more anterior hippocampal activation during encoding of visual associations. We therefore conclude that the degree to which anterior parts of the hippocampus are activated during initial learning is correlated with the special characteristics of the stimuli. With respect to the neural activity during maximum recall, the same arguments apply. It was assumed that processing of multiple relational stimuli should produce similar anterior MTL activations as during initial encoding (Schacter and Wagner 1999). This is substantiated empirically by our results and a previous study demonstrating anterior hippocampal activity when comparing the neural activity during recall of spatial relations between objects with the activity during the recall of locational information per se (Owen et al. 1996). We conclude that the extent of functional segregation of hippocampal functions is determined not only by the aspects of novelty and memorability during encoding and recall but also by the specific characteristics of the material to be memorized requiring the establishment of intra-item associations.

\section{Delayed Recall}

While we observed hippocampal activity during initial and maximum recall, no hippocampal activity was found during delayed recall even when lowering statistical thresholds. Thus, the present data may correspond to traditional views of a temporary role of the hippocampus in retrieving previously learned information (Alvarez and Squire 1994; Squire and Alvarez 1995; Schacter et al. 1996). However, this is only a very tentative explanation for the nonobservance of hippocampal activity during delayed recall because of the rather short delay between last presentation and the delayed recall condition that was due to technical restrictions. In fact, graded retrograde amnesia can extend across several months and years (Milner et al. 1998) suggesting that medial temporal structures contribute to consolidation over a prolonged time period. However, clinical data from dementia research clearly indicate the importance of an early critical time interval in the first few minutes in which abnormal forgetting of information occurs, that is, information cannot be sufficiently consolidated by these patients (Carlesimo et al. 1993).

Comparing neural activity during initial and maximum recall with that during delayed recall, it can be summarized that a transition of neural activity was observed from posterior (inferior occipital, parietal) to more anterior (temporal) cortical regions (Table 1 and Fig. 4B). This observation closely parallels a transition from the dorsal to the ventral processing stream, or, in functional terms, from spatially- based information processing to object-oriented information processing (Ungerleider and Haxby 1994). The following mechanism may be reflected by our data. Learning of the patterns can functionally be subdivided in memorizing the constituting items and the associations between them. Therefore, the anterior hippocampal activity during recall functionally reflects a process of re-establishing the associations between the constituting single rectangles. When both information sources are bound such that an image emerges or a "Gestalt" forms, this functional transition is reflected by an increasing activity of the lateral temporal cortices. This means that the more the information is combined the less the dorsal processing is engaged and the more the ventral processing stream is involved. Thus, while hippocampal activity at maximum recall in the present study is still needed for the associative recombination of the patterns, at delayed recall hippocampal activity is no longer needed. Additionally, activation of the superior parietal regions is reduced, and activity of the temporal cortices is increased. Thus, the temporal cortices are now the storehouse of long-term information which is in good accordance with a series of studies on semantic, but not episodic, memory (Zola-Morgan and Squire 1993; Miyashita 1993, 1998; Graham et al. 1999; Tomita et al. 1999).

In summary, the present task, which was designed analogous to classic list-learning paradigms, allows systematic evaluation of encoding and active recall under the constraints imposed by simultaneous observation with fMRI. We conclude that both lateralization and segregation of hippocampal activity depend on the specific characteristics of the stimuli requiring the establishment of intra-item associations for encoding as well as for retrieval. Although the material was visual in nature, basic linguistic processing concerning the formation of those associations may contribute to left-sided lateralization. The absence of hippocampal activation during delayed recall together with the increase of lateral temporal involvement possibly corresponds with an emerging transition from episodic to long-term memory.

\section{MATERIALS AND METHODS}

\section{Subjects}

Twelve healthy young subjects (six males, six females; age $26.1 \pm 2.1 \mathrm{yr}$; means $\pm \mathrm{SD}$ ) gave informed consent before the study. All subjects were right-handed according to the Oldfield (Oldfield 1971 ) handiness quotients (range: +87 to +100 ). None had any sign of color blindness or visual field defects. The medical history was taken from each individual, and the study was approved by the local ethics committee.

\section{MRI Data Acquisition}

Data were acquired with a 1.5 Tesla Magnetom VISION (Siemens) whole-body MRI system equipped with a head volume coil. $\mathrm{T}_{2}{ }^{*}$ weighted functional MR images were obtained using echo-planar imaging in axial orientation ( $\mathrm{TE}=66 \mathrm{~ms} ; 121.9 \mathrm{~ms}$ per image). Image size was $64 \times 64$ pixels $(3.6 \times 3.6 \mathrm{~mm}$ pixels $)$. The volume

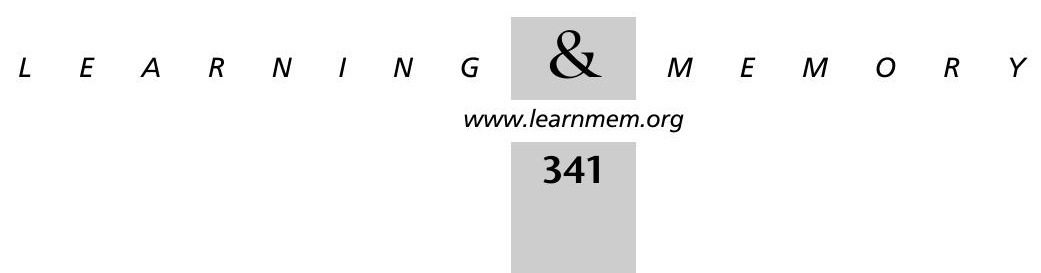


consisted of 32 slices $(\mathrm{TR}=4000 \mathrm{~ms}$ ). Slice thickness was $3 \mathrm{~mm}$ with a gap of $0.6 \mathrm{~mm}$. Thus, voxel size was isotropic. For anatomical reference $\mathrm{T}_{2}$-weighted turbo-spin-echo images $(256 \times 256 ; 32$ slices; voxel-size: $0.9 \times 0.9 \times 3.6 \mathrm{~mm}$ ) were obtained. The first six volumes of each session were discarded to allow for $\mathrm{T}_{1}$ equilibration effects.

\section{fMRI Memory Task}

The new paradigm requires repetitive memorization of geometric patterns and repetitive active recall in an fMRI-compatible manner (see Fig. 5A). Except for stimulus qualities, the paradigm is analogous to behaviorally well-established verbal learning tests, such as the California Verbal Learning Test (Lezak 1995). Repetitive listlearning paradigms represent one of the prototype test situations for neuropsychological assessment of the integrity of episodic memory functions. Because of the repetitions, and consequently the availability of an individual learning gradient, such paradigms are well suited to assess neural processing related to encoding and

A

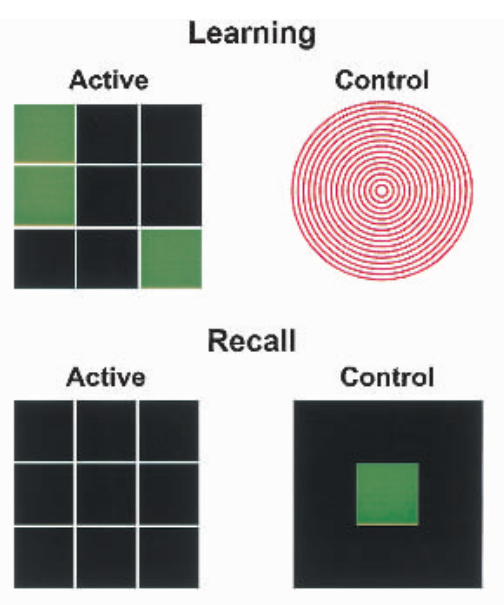

B

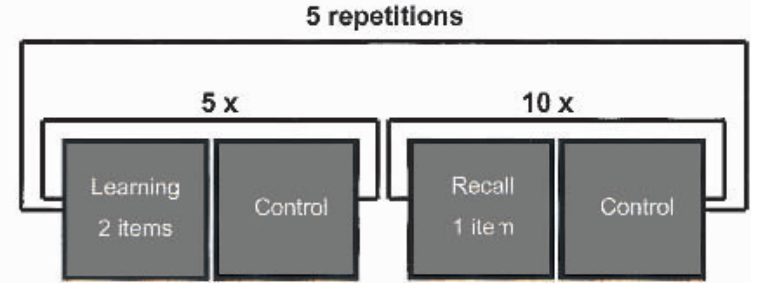

Figure 5 The Memory Paradigm. (A) A typical example of one of 10 abstract geometric patterns (left upper panel). All items were constructed by means of green colored patterns within a $3 \times 3$ matrix. A figure of red concentric circles (right upper panel) was the stimulus during the off-cycle of the learning session. Upon completion of memorization, the subjects were shown the empty matrix (left lower panel). The patterns could easily be reproduced by a specially designed fMRI-compatible keyboard consisting of $3 \times 3$ buttons. As the control condition during recall, a single reaction task was inserted (right lower panel). A green rectangle appeared in the center of the screen. Subjects had to respond by pressing the middle button of the keyboard as fast as possible after appearance. (B) A schematic overview of the stimulation protocol (see text). recollection of items at decreasing levels of novelty. This can be achieved by contrasting neural activity of initial encoding or retrieval against their corresponding later phases (and vice versa) in which stimuli have become more easily memorized as indicated by individual performance gain. Additionally, assessments of neural activity related to novelty and memorability can be performed within the same processing domains of encoding and retrieval. Selection of the kind of stimulus material was motivated by considerations with respect to practicability for the subject to actively recall and compatibility with fMRI requirements. More specifically, stimuli should allow for the establishment of intra-item associations. Intra-item associations are strong for various types of visual stimuli, for example, landscapes or faces. However, such items may be completely recollected by filling in missing parts even if not memorized. Our material substantially differs in that recall of parts of the pattern does not suffice to compensate for parts not memorized. Thus, more so for the present stimuli than, for example, face drawings, associations must be set up to successfully encode and retrieve the stimuli; that is, for a complete learning the stimuli automatically enforce the subject to build up the associations, whereas, for example, a landscape can be retrieved only by one or more details.

A list of 10 abstract geometric patterns was presented during a learning condition via LCD goggles. The subjects saw a pattern of green rectangles appearing within a $3 \times 3$ black matrix with white grid lines via LCD goggles (Fig. 5A, left upper panel). Within one on-cycle, two figures were sequentially presented for a duration of $6 \mathrm{sec}$. The total length of one on-cycle was $12 \mathrm{sec}$ ( 3 TRs). During the following off-cycle, a figure consisting of red circles (Fig. 5A, right upper panel) was shown for a duration of $20 \mathrm{sec}(5 \mathrm{TRs})$. Then, the next two memory items were shown, followed by an off-cycle and so on. To present the 10 memory items, there were five on-cycles during the learning session (Fig. 5B). The learning condition was immediately followed by a recall condition. Subjects saw an empty $3 \times 3$ black matrix with white grid lines via the LCD goggles. The matrix could be filled by pressing the corresponding buttons of a keyboard to reproduce the pattern of rectangles seen during learning (Fig. 5A left lower panel). Subjects could not see the keyboard itself during scanning and were familiarized with the keyboard before scanning. The recall session comprised 10 on/offcycles of the same duration as those during scanning or learning (Fig. 5B). One memorized pattern could be reproduced during each on-cycle. The subjects were allowed to choose the order of recall and to correct their input within the time interval of one on-cycle. At the end of the on-cycle the responses of the subjects were automatically registered by a personal computer (PC). During the subsequent off-cycle a single green rectangle appeared on the center of the screen. The background was black and the white grid was not shown (Fig. 5A, right lower panel). The subjects had to respond to the stimulus by pressing the middle button of the keyboard with their right index fingers. Thus, hand and finger position were standardized before beginning the next on-cycle. The mean presentation rate of the green rectangle was paralleled to the mean number of button presses to correctly recall the patterns. The presentation of the rectangle was at random time intervals. Altogether, learning and recall phases were repeated five times. Neither the items nor the order of presentation were altered across the five learning conditions. About $15 \mathrm{~min}$ after the fifth recall condition, subjects were scanned again during a sixth delayed recall condition.

The paradigm was programmed with Turbo Pascal 6.0 (Borland's, Inc.) on a standard PC. Signal transduction of the button presses to the PC was realized by means of a fiber-optic system and

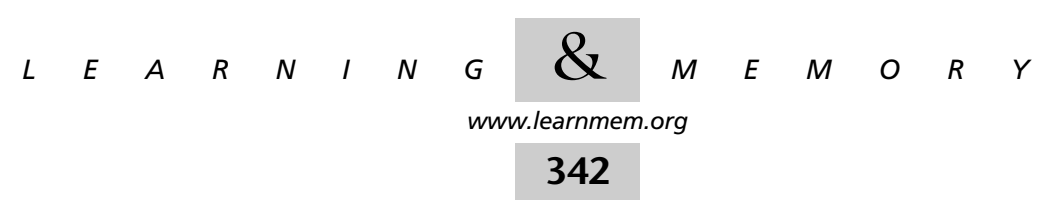


an opto-coupler. The keyboard had nine buttons, each corresponding to the respective element of the $3 \times 3$ matrix. Within the scanner, items and the recall matrices were presented by means of LCD video-goggles (Resonance Technology). Onset of the PC-stimulation epochs was controlled by the fMRI scanner emitting a TTL pulse before each volume acquisition.

\section{fMRI Data Analysis}

Image preprocessing and statistical analysis were performed with Statistical Parametric Mapping (SPM99, Wellcome Department of Cognitive Neurology; http://www.fil.ion.ucl.ac.uk) executed in MATLAB 5.3 (The MathWorks). All individual functional images were corrected for motion artifacts by realignment to the first volume of the session. A sinc interpolation was used to reslice the realigned volumes. A $\mathrm{T}_{2}$-weighted structural MRI was coregistered to the mean image of the realigned volumes. All images were spatially normalized to a standard template of $2 \times 2 \times 2 \mathrm{~mm}$ voxels. Finally, images were spatially smoothed with a $10-\mathrm{mm}$ full width half maximal (FWHM) isotropic Gaussian kernel. For each condition the variance of each and every voxel was estimated according to the general linear model using a box-car model convoluted with the hemodynamic response function as the predictor. Images were adjusted for global effects and low frequency drifts were removed via a high-pass filter using low-frequency cosine functions with a cutoff of $72 \mathrm{sec}$. Individual regionally specific effects between the active and resting phases were computed using linear contrasts. For each subject the appropriate contrast images were calculated using directed single-tailed $t$-tests producing a $t$ statistic for each voxel. To account for inter-individual variance, all group analyses were computed using a Random Effects Model. Group analysis across subjects was performed by means of one-sample $t$-tests on the resulting images of the interesting contrasts of each individual subject. For Random Effects Analysis, voxels and clusters of significant voxels were thresholded at a $P$-level of $P<.01$, uncorrected for multiple comparisons. At the cluster-level, significance was set to an uncorrected $p$-level of $p<.05$. Areas were labeled using the nomenclature of Talairach and Tournoux (1988) and Brodmann (1909).

Because we were most interested in hippocampal activity, we assumed that activation of the hippocampus should be best detectable with respect to novelty processing (e.g., Parkin 1997; Menon et al. 2000) and high memorability (e.g., ; Squire et al. 1992; Cameron et al. 2001) during encoding and retrieval, respectively. Therefore, the following planned contrasts were calculated for each individual. For assessment of high memorability processing, neural activity during the phase of individual maximum recall was contrasted against the very first recall phase (maximum recall minus first recall). Neural activity at a level of low memorability was assessed by inverting this contrast (first recall minus maximum recall). To calculate neural activations due to encoding of novel items, activity during the first learning phase was contrasted against the learning phase that was before the phase of individual maximum recall (initial learning minus late learning). Accordingly, low novelty encoding was analyzed by inverting the contrast (late learning minus initial learning). This contrast was only computed for reasons of completeness and exploration. Although all other contrasts are not subject to possible mutual influences of learning and retrieval, activity during late learning may be influenced by processes of retrieval that take place during presentation of the items. Therefore, within this contrast processes cannot be clearly disentangled and results are only of exploratory value. Cross-compari- sons between learning and recall were also not computed to exclude misinterpretations of the novelty/memorability assessments that may be due to general differences in processing demands for encoding and retrieval. Finally, to find out the relevant neural substrate during delayed recall, neural activity during the sixth recall phase was contrasted against neural activity during the first recall phase (delayed recall minus first recall phase). All individual results of the corresponding contrast analyses were passed to separate random effects analysis to obtain results for the whole group.

The publication costs of this article were defrayed in part by payment of page charges. This article must therefore be hereby marked "advertisement" in accordance with 18 USC section 1734 solely to indicate this fact.

\section{REFERENCES}

Aggleton, J.P. and Brown, M.W. 1999. Episodic memory, amnesia, and the hippocampal-anterior thalamic axis. Behav. Brain Sci. 22: 425-489.

Aguirre, G.K., Detre, J.A., Alsop, D.C., and D'Esposito, M. 1996. The parahippocampus subserves topographical learning in man. Cereb. Cortex 6: 823-829.

Alvarez P. and Squire L.R. 1994. Memory consolidation and the medial temporal lobes: A simple network model. Proc. Natl. Acad. Sci. 91: 7041-7045.

Bohbot, V.D., Kalina, M., Stepankova, K., Spackova, N., Petrides, M., and Nadel, L. 1998. Spatial memory deficits in patients with lesions to the right hippocampus and to the right parahippocampal cortex. Neuropsychologia 36: 1217-1238.

Brewer, J.B., Zhao, Z., Desmond, J.E., Glover, G.H., and Gabrieli, J.D. 1998. Making memories: Brain activity that predicts how well visual experience will be remembered. Science 281: 1185-1187.

Brodmann, K. 1909. Vergleichende Lokalisationslebre der Großbirnrinde Barth, Leipzig.

Buckner, R.L. and Koutstaal, W. 1998. Functional neuroimaging studies of encoding, priming, and explicit memory retrieval. Proc. Natl. Acad. Sci. 95: 891-898.

Buckner, R.L., Petersen, S.E., Ojemann, J.G., Miezin, F.M., Squire, L.R., and Raichle, M.E. 1995. Functional anatomical studies of explicit and implicit memory retrieval tasks. J. Neurosci. 15: 12-29.

Cabeza, R., Anderson, N.D., Houle, S., Mangels, J.A., and Nyberg, L. 2000 Age-related differences in neural activity during item and temporal-order memory retrieval: A positron emission tomography study. J. Cogn. Neurosci. 12: 197-206.

Cameron K.A., Yashar S., Wilson C.L., and Fried, I. 2001. Human hippocampal neurons predict how well word pairs will be remembered. Neuron 30: 289-298.

Carlesimo, G.A., Fadda, L., Bonci, A., and Caltagirone, C. 1993. Differential rates of forgetting from long-term memory in Alzheimer's and multi-infarct dementia. Int. J. Neurosci. 73: 1-11.

Cohen, N.J., Ryan, J., Hunt, C., Romine, L., Wszalek, T., and Nash, C. 1999. Hippocampal system and declarative (relational) memory: Summarizing the data from functional neuroimaging studies. Hippocampus 9: 83-98.

Corkin, S., Amaral, D.G., Gonzalez, R.G., Johnson, K.A., and Hyman, B.T. 1997. H. M.'s medial temporal lobe lesion: Findings from magnetic resonance imaging. J. Neurosci. 17: 3964-3979.

Dobbins, I.G., Kroll, N.E., Tulving, E., Knight, R.T., and Gazzaniga, M.S 1998. Unilateral medial temporal lobe memory impairment: Type deficit, function deficit, or both? Neuropsychologia 36: 115-127.

Dolan, R.J. and Fletcher, P.F. 1999 Encoding and retrieval in human medial temporal lobes: An empirical investigation using functional magnetic resonance imaging (fMRI). Hippocampus 9: 25-34.

Eldridge, L.L., Knowlton, B.J., Furmanski, C.S., Bookheimer, S.Y., and Engel, S.A. 2000. Remembering episodes: A selective role for the hippocampus during retrieval. Nat. Neurosci. 3: 1149-1152.

Fernandez, G., Weyerts, H., Schrader-Bolsche, M., Tendolkar, I., Smid, H.G., Tempelmann, C., Hinrichs, H., Scheich, H., Elger, C.E., Mangun,

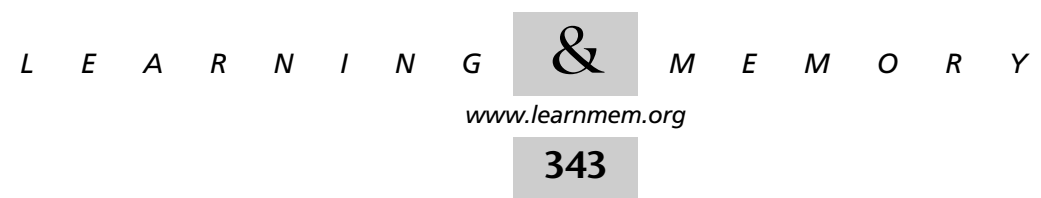


G.R., et al.. 1998. Successful verbal encoding into episodic memory engages the posterior hippocampus: A parametrically analyzed functional magnetic resonance imaging study. J. Neurosci. 18: 1841-1847.

Fletcher, P.C., Frith, C.D., Grasby, P.M., Shallice, T., Frackowiak, R.S., and Dolan, R.J. 1995. Brain systems for encoding and retrieval of auditory-verbal memory. An in vivo study in humans. Brain 118: $401-416$.

Gabrieli, J.D.E., Brewer, J.B., Desmond, J.E., and Glover, G.H. 1997. Separate neural bases of two fundamental memory processes in the human medial temporal lobe. Science 276: 264-266.

Graham, K.S. and Hodges, J.R. 1997. Differentiating the roles of the hippocampal complex and the neocortex in long-term memory storage: Evidence from the study of semantic dementia and Alzheimer's disease. Neuropsychology 11: 77-89.

Graham, K.S., Patterson, K., and Hodges, J.R. 1999. Episodic memory: New insights from the study of semantic dementia. Curr. Opin. Neurobiol. 9: 245-250.

Grön, G., Wunderlich, A.P., Spitzer, M., Tomczak, R., and Riepe, M.W 2000. Human brain activation during navigation: Gender-dependent neural networks as substrate of performance. Nat. Neurosci. 3: 404-408.

Hamilton, C.R. and Vermeire, B.A. 1988. Complementary hemispheric specialization in monkeys. Science 242: 1691-1694.

Henson, R.N., Rugg, M.D., Shallice, T., Josephs, O., and Dolan, R.J. 1999. Recollection and familiarity in recognition memory: An event-related functional magnetic resonance imaging study. J. Neurosci. 19: 3962-3972

Jones-Gotman, M., Zatorre, R.J., Olivier, A., Andermann, F., Cendes, F., Staunton, H., McMackin, D., Siegel, A.M., and Wieser, H.G. 1997. Learning and retention of words and designs following excision from medial or lateral temporal-lobe structures. Neuropsychologia 35: 963-973.

Kelley, W.M., Miezin, F.M., McDermott, K.B., Buckner, R.L., Raichle, M.E., Cohen, N.J., Ollinger, J.M., Akbudak, E., Conturo, T.E., Snyder, A.Z., et al. 1998. Hemispheric specialization in human dorsal frontal cortex and medial temporal lobe for verbal and nonverbal memory encoding. Neuron 20: 927-936

Kosslyn, S.M., Koenig, O., Barrett, A., Cave, C.B., Tang, J., and Gabrieli, J.D. 1989. Evidence for two types of spatial representations: Hemispheric specialization for categorical and coordinate relations. $J$. Exp. Psychol. Hum. Percept Perform. 15: 723-735.

Lepage, M., Habib, R., and Tulving, E. 1998. Hippocampal PET activations of memory encoding and retrieval: The HIPER model. Hippocampus 8: 313-322.

Lezak, M. 1995. Neuropsychological Assessment, 3rd ed, pp. 445-448. Oxford University Press, New York, Oxford.

Maguire, E.A., Frackowiak, R.S., and Frith, C.D. 1996. Learning to find your way: A role for the human hippocampal formation. Proc. R. Soc. Lond. B. Biol. Sci. 263: 1745-1750.

Martin, A. 1999. Automatic activation of the medial temporal lobe during encoding: Lateralized influences of meaning and novelty. Hippocampus, 9: 62-70.

Menon, V., White, C.D., Eliez, S., Glover, G.H., and Reiss, A.L. 2000. Analysis of a distributed neural system involved in spatial information, novelty, and memory processing. Hum. Brain Mapp. 11: 117-129.

Milner, B., Squire, L.R., and Kandel, E.R. 1998. Cognitive neuroscience and the study of memory. Neuron 20: 445-468.

Miyashita, Y. 1988. Neuronal correlate of visual associative long-term memory in the primate temporal cortex. Nature 335: 817-820.

Miyashita, Y. 1993. Inferior temporal cortex: Where visual perception meets memory. Annu. Rev. Neurosci 16: 245-263.

Montaldi, D., Mayes, A.R., Barnes, A., Pirie, H., Hadley, D.M., Patterson, J., and Wyper, D.J. 1998. Associative encoding of pictures activates the medial temporal lobes. Hum. Brain Mapp. 6: 85-104.

Moser, M.B. and Moser, E.I. 1998. Distributed encoding and retrieval of spatial memory in the hippocampus. J. Neurosci. 18: 7535-7542.

Nadel, L. and Moscovitch, M. 1997. Memory consolidation, retrograde amnesia and the hippocampal complex. Curr. Opin. Neurobiol. 7: 217-227.

Nadel, L., Samsonovich, A., Ryan, L., and Moscovitch, M. 2000. Multiple trace theory of human memory: computational, neuroimaging, and neuropsychological results. Hippocampus 10: 352-368.

Oldfield, R.C. 1971. The assessment and analysis of handedness: The Edinburgh inventory. Neuropsychologia 9: 97-113.

Owen, A.M., Milner, B., Petrides, M., and Evans, A.C. 1996. Memory for object features versus memory for object location: A positron-emission tomography study of encoding and retrieval processes. Proc. Natl. Acad. Sci. 93: 9212-9217.

Parkin A.J. 1997. Human memory: Novelty, association and the brain. Curr. Biol. 7: 768-769.

Paulesu, E., Frith, C.D., and Frackowiak, R.S. 1993. The neural correlates of the verbal component of working memory. Nature 362: 342-345.

Paulesu, E., Frith, U., Snowling, M., Gallagher, A., Morton, J., Frackowiak, R.S., and Frith, C.D. 1996. Is developmental dyslexia a disconnection syndrome? Evidence from PET scanning. Brain 119: 143-157.

Phelps, E.A., Hirst, W., and Gazzaniga, M.S. 1991. Deficits in recall following partial and complete commissurotomy. Cereb. Cortex 1: $492-498$.

Pruessner, J.C., Li, L.M., Serles, W., Pruessner, M., Collins, D.L., Kabani, N., Lupien, S., and Evans, A.C. 2000. Volumetry of hippocampus and amygdala with high-resolution MRI and three-dimensional analysis software: Minimizing the discrepancies between laboratories. Cereb Cortex 10: 433-442.

Roland, P.E. and Gulyas, B. 1995. Visual memory, visual imagery, and visual recognition of large field patterns by the human brain: Functional anatomy by positron emission tomography. Cereb. Cortex 5: 79-93.

Rombouts, S.A., Machielsen, W.C., Witter, M.P., Barkhof, F., Lindeboom, J., and Scheltens, P. 1997. Visual association encoding activates the medial temporal lobe: A functional magnetic resonance imaging study. Hippocampus 7: 594-601.

Saykin, A.J., Johnson, S.C., Flashman, L.A., McAllister, T.W., Sparling, M., Darcey, T.M., Moritz, C.H., Guerin, S.J., Weaver, J., and Mamourian, A. 1999. Functional differentiation of medial temporal and frontal regions involved in processing novel and familiar words: An fMRI study. Brain 122: 1963-1971

Schacter, D.L., Alpert, N.M., Savage, C.R., Rauch, S.L., and Albert, M.S. 1996. Conscious recollection and the human hippocampal formation: Evidence from positron emission tomography. Proc. Natl. Acad. Sci. 93: 321-325.

Schacter, D.L., Curran, T., Reiman, E.M., Chen, K., Bandy, D.J., and Frost, J.T. 1999. Medial temporal lobe activation during episodic encoding and retrieval: A PET study. Hippocampus 9: 575-581.

Schacter, D.L. and Wagner, A.D. 1999. Medial temporal lobe activations in fMRI and PET studies of episodic encoding and retrieval. Hippocampus 9: 7-24.

Sergent, J. 1991. Processing of spatial relations within and between the disconnected cerebral hemispheres. Brain 114: 1025-1043.

Shallice, T., Fletcher, P., Frith, C.D., Grasby, P., Frackowiak, R.S., and Dolan, R.J. 1994. Brain regions associated with acquisition and retrieval of verbal episodic memory. Nature 368: 633-635.

Stark, C.E. and Squire, L.R. 2000. Functional magnetic resonance imaging (fMRI) activity in the hippocampal region during recognition memory J. Neurosci., 20: 7776-7781.

Squire, L.R., Ojemann, J.G., Miezin, F.M., Petersen, S.E., Videen, T.O., and Raichle, M.E. 1992. Activation of the hippocampus in normal humans: A functional anatomical study of memory. Proc. Natl. Acad. Sci. 89: $1837-1841$.

Squire, L.R. and Alvarez, P. 1995. Retrograde amnesia and memory consolidation: A neurobiological perspective. Curr. Opin. Neurobiol. 5: 169-177.

Stern, C.E., Corkin, S., Gonzalez, R.G., Guimaraes, A.R., Baker, J.R., Jennings, P.J., Carr, C.A., Sugiura, R.M., Vedantham, V., and Rosen, B.R. 1996. The hippocampal formation participates in novel picture

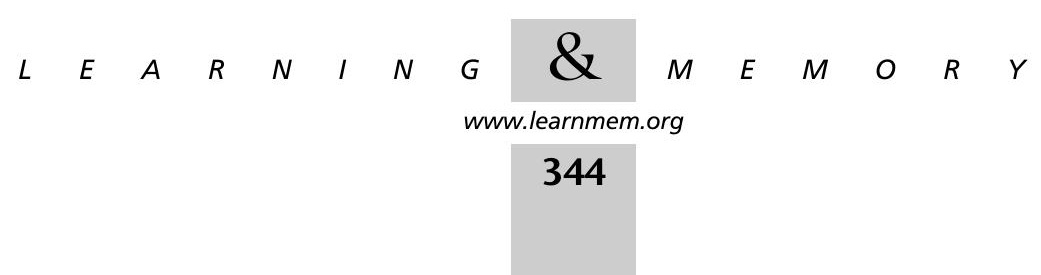


encoding: Evidence from functional magnetic resonance imaging. Proc. Natl. Acad. Sci. 93: 8660-8665.

Talairach, J. and Tournoux, P. 1988. Co-planar stereotaxic atlas of the buman brain. Georg-Thieme Verlag, Stuttgart.

Tomita, H., Ohbayashi, M., Nakahara, K., Hasegawa, I., and Miyashita, Y. 1999. Top-down signal from prefrontal cortex in executive control of memory retrieval. Nature 401: 699-703.

Ungerleider, L.G. and Haxby, J.V. 1994. 'What' and 'where' in the human brain. Curr. Opin. Neurobiol. 4: 157-165.

Vargha-Khadem, F., Gadian, D.G., Watkins, K.E., Connelly, A., Van Paesschen, W., and Mishkin, M. 1997. Differential effects of early hippocampal pathology on episodic and semantic memory. Science 277: 376-380

Wood, E.R., Dudchenko, P.A., and Eichenbaum, H. 1999. The global record of memory in hippocampal neuronal activity. Nature 397: 613-616.

Zola-Morgan, S. and Squire, L.R. 1993. Neuroanatomy of memory. Annu. Rev. Neurosci. 16: 547-563.

Received June 29, 2001; accepted in revised form October 22, 2001.

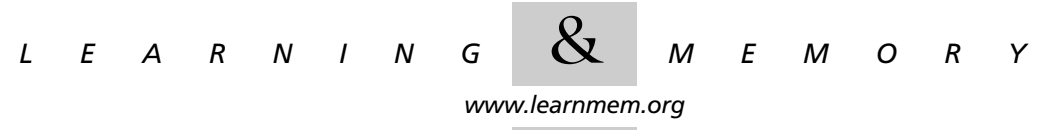




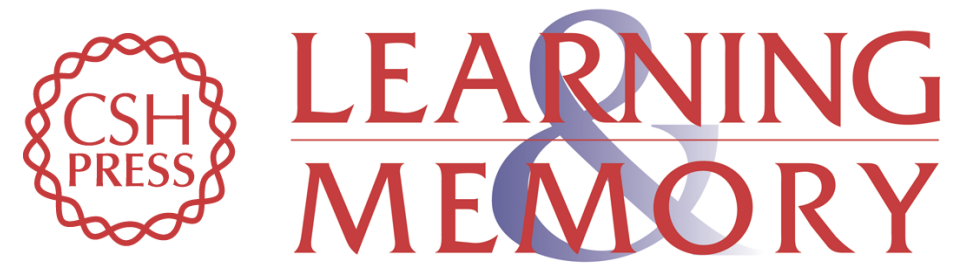

\section{Hippocampal Activations during Repetitive Learning and Recall of Geometric Patterns}

Georg Grön, Daniel Bittner, Bernd Schmitz, et al.

Learn. Mem. 2001, 8:

Access the most recent version at doi:10.1101//m.42901

References This article cites 61 articles, 16 of which can be accessed free at: http://learnmem.cshlp.org/content/8/6/336.full.html\#ref-list-1

License

Email Alerting Receive free email alerts when new articles cite this article - sign up in the box at the Service top right corner of the article or click here. 